\title{
The Rate of Falloff of Ising Model Correlations at Large Temperature
}

\author{
Barry Simon ${ }^{1,2}$
}

Received November 14, 1980

We recover results of Abraham and Kunz and Paes-Leme on falloff of Ising model correlations at high temperature by using nothing more than hightemperature diagrams.

KEY WORDS: Ising model; high temperature; correlation delay.

\section{INTRODUCTION}

In this paper, we want to consider the nearest-neighbor $\nu$-dimensional Ising ferromagnet at inverse temperatures $\beta$ (with Hamiltonian normalized by each neighbor pair counted exactly once in $H$ ). There has been some interest in determining the leading behavior of the rate of falloff of the two-point function which has rather complicated directional dependence because the symmetry of the lattice is only discrete. Camp and Fisher ${ }^{(2,3)}$ found the behavior using formal arguments and subsequently Abraham and $\mathrm{Kunz}^{(1)}$ and Paes-Leme ${ }^{(5)}$ found rigorous proofs. Let $\omega \in S^{\nu-1}$ the unit sphere and define

$$
m(\omega, \beta)=\varlimsup_{\substack{\|\alpha\| \rightarrow \infty \\ \alpha /\|\alpha\| \rightarrow \omega}}-\|\alpha\|^{-1} \ln \left\langle\sigma_{\alpha} \sigma_{0}\right\rangle_{\beta}
$$

where $\langle\cdot\rangle_{\beta}$ is the state at inverse temperature $\beta$ (unique for $\beta$ small).

\footnotetext{
Research partially supported by NSF Grant MCS-78-01885.

${ }^{1}$ Department of Mathematics, California Institute of Technology. On leave from Departments of Mathematics and Physics, Princeton University.

${ }^{2}$ Sherman Fairchild Visiting Scholar.
} 
Letting $\omega_{j}$ be the components of $\omega$, Paes-Leme proves

$$
m=(-\ln \beta) \sum_{1}^{\nu}\left|\omega_{j}\right|+\sum_{1}^{\nu}\left|\omega_{j}\right| \ln \left|\omega_{j}\right|-\left[\sum_{1}^{\nu}\left|\omega_{j}\right| \ln \left(\sum_{1}^{\nu}\left|\omega_{j}\right|\right)\right]+O(\beta)
$$

All the above papers depend on analysis of direct correlation functions and analyticity arguments. Our purpose here is to give a simple proof of (1) in terms of elementary high-temperature diagrams. The point is that for fixed $\alpha=\left(\alpha_{1}, \ldots, \alpha_{\nu}\right)$, the leading term as $\beta \downarrow 0$ is (if $\alpha_{i} \geqslant 0$ )

$$
L(\alpha, \beta)=\frac{\left(\alpha_{1}+\alpha_{2}+\cdots+\alpha_{v}\right) !}{\alpha_{1} ! \cdots \alpha_{\nu} !}(\tanh \beta)^{\alpha_{1}+\cdots+\alpha_{p}}
$$

If one uses Stirling's formula, one sees that

$$
\begin{aligned}
\lim _{\substack{\alpha\left|\rightarrow \infty \\
\alpha_{i} /\right| \alpha \mid \rightarrow \omega_{i}}}-|\alpha|^{-1} L(\alpha, \beta)= & \ln (\tanh \beta) \sum_{1}^{\nu}\left|\omega_{j}\right| \\
& +\sum_{1}^{\nu}\left|\omega_{j}\right| \ln \left(\left|\omega_{j}\right| /\left|\omega_{1}\right|+\cdots+\left|\omega_{\nu}\right|\right)
\end{aligned}
$$

Thus (1) just says that for the mass falloff, one can, in a sense, interchange the $\beta \rightarrow 0$ and the $\alpha \rightarrow \infty$ limits. Using diagrammatic ideas, we will prove upper and lower bounds that rigorously justify this. In Section 2 , we prove that

$$
\left\langle\sigma_{0} \sigma_{\alpha}\right\rangle_{\beta} \geqslant L(\alpha, \beta) \exp \left[-c \beta\left(1+\sum_{1}^{p}\left|\alpha_{i}\right|\right)\right]
$$

for some $c$ and all $\alpha, \beta$. In Section 3, we prove that for $\beta$ sufficiently small and $|\alpha|$ sufficiently large

$$
\left\langle\sigma_{0} \sigma_{\alpha}\right\rangle_{\beta} \leqslant 3 L(\alpha, \beta)(1+d \beta)^{\sum_{i}^{i}\left|\alpha_{i}\right|}
$$

for a constant $d$. (3), (4), and (5) immediately prove (1). bution.

In Section 4, we discuss extensions including general even spin distri-

\section{LOWER BOUNDS FOR SPIN-1/2}

Writing

$$
\exp \left(\beta \sigma_{i} \sigma_{j}\right)=\cosh \beta\left(1+\sigma_{i} \sigma_{j} \tanh \beta\right)
$$

[since $\left(\sigma_{i} \sigma_{j}\right)^{2}=1$ ], we see that for any volume $\Lambda$

$$
\left\langle\sigma_{0} \sigma_{\alpha}\right\rangle_{\Lambda, \beta}=\frac{\left\langle\sigma_{0} \sigma_{\alpha} \prod_{\langle i j\rangle}\left(1+\sigma_{i} \sigma_{j} \tanh \beta\right)\right\rangle_{0}}{\left\langle\prod_{\langle i j\rangle}\left(1+\sigma_{i} \sigma_{j} \tanh \beta\right)\right\rangle_{0}}
$$

and in the usual way (see, e.g., Ref. 4), we extend the product and associate 
terms with graphs. A graph consists of some sets of nearest-neighbor bounds. The boundary of a graph is the set of sites where an odd number of bounds occur in the graph. In the denominator of (6b), only graphs with no boundary count and in the numerator only graphs with boundary $\{0, \alpha\}$ enter. The value of a graph is $(\tanh \beta)^{n}$ with $n$ the number of bounds in the graph.

Two features of these graphs are critical:

(a) All values are positive.

(b) The value of a graph is the product of the values of its connected components.

To get the lower bound, only keep the graphs in the numerator with the property that they have as a connected component, one of the

$$
N=\left(\alpha_{1}+\cdots+\alpha_{v}\right) ! / \alpha_{1} ! \cdots \alpha_{v} !
$$

graphs of length $\alpha_{1}+\cdots+\alpha_{v}$ which join 0 and $\alpha$ (this is the number since among the $\alpha_{1}+\cdots+\alpha_{v}$ steps one must choose $\alpha_{1}$ going in the 1direction, etc.). Label these graphs $\gamma_{1}, \ldots, \gamma_{N}$. Thus, the set of graphs discussed gives a contribution

$$
\left\langle\sigma_{0} \sigma_{\alpha}\right\rangle_{\Lambda, \beta} \geqslant \sum_{i=1}^{N}(\tanh \beta)^{\sum \alpha_{i}} Z\left(\gamma_{i}\right) / Z
$$

where $Z$ is the denominator of (6b) and $Z\left(\gamma_{i}\right)$ is the set of graphs disjoint from $\gamma_{i}$. But $Z\left(\gamma_{i}\right)$ is exactly the partition function for a model with all interactions of spins in $\gamma_{i}$ dropped, i.e.,

$$
\begin{aligned}
Z\left(\gamma_{i}\right) / Z & =\left\langle\left[\prod_{\langle k l\rangle}^{(i)}\left(1+\sigma_{k} \sigma_{l} \tanh \beta\right)\right]^{-1}\right\rangle_{\Lambda, \beta} \\
& =\left\langle\left[\prod_{\langle k l\rangle}^{(i)} e^{-\beta \sigma_{k} \sigma_{l}} \cosh \beta\right]\right\rangle_{\Lambda, \beta} \\
& \geqslant\left\langle\exp \left(-\sum_{\langle k l\rangle}^{(i)} \beta \sigma_{k} \sigma_{l}\right)\right\rangle_{\Lambda, \beta} \\
& \geqslant \exp \left[-\beta\left\langle\sum_{\langle k l\rangle}^{(i)} \sigma_{k} \sigma_{l}\right\rangle_{\Lambda, \beta}\right] \\
& \geqslant \exp \left\{-\beta(2 v)\left[1+\left(\sum_{i}^{\nu} \alpha_{i}\right)\right]\right\}
\end{aligned}
$$

where $\Pi^{(i)}, \Sigma^{(i)}$ denotes the product and sum over all neighbor pairs $\langle k l\rangle$ 
with either $k \in \gamma_{i}$ or $l \in \gamma_{i}$. (8) comes from $\cosh \beta>1$, (9) from Jensen's inequality, and (10) from a crude estimate of the number of spins interacting with $\gamma_{i}$.

\section{UPPER BOUNDS FOR SPIN-1/2}

We begin by recalling Fisher's upper bound ${ }^{(4)}$ : Let $N(\alpha, k)$ be the number of noninteracting random walks of $k$ steps from 0 to $\alpha$. Then

$$
\left\langle\sigma_{\alpha} \sigma_{0}\right\rangle_{\beta} \leqslant \sum_{k} N(\alpha, k)[\tanh \beta]^{k}
$$

This follows from the analysis of the last section if we note that $Z(\gamma) / Z \leqslant 1$ for any $\gamma$ (and if we note that one need not only deal with connected graphs but can "factor out" any graph with no boundary; actually, below we do not use the nonintersecting property).

Let

$$
n=\sum \alpha_{i}
$$

so that

$$
L(\alpha, \beta)=N(\alpha, n)[\tanh \beta]^{n}
$$

We just need to bound $N(\alpha, k)$. Only $k$ 's of the form $n+2 l$ have nonzero $N$. One has the trivial bound

$$
N(\alpha, n+2 l) \leqslant(2 v-1)^{n+2 l}
$$

coming from counting all random walks without self-intersection whether they end at $\alpha$ or not. This bound is fine for large $l$ but for small $l$ it is too much bigger than $N(\alpha, n)$.

Any path, $\gamma$, from 0 to $\alpha$ with $n+2 l$ steps has precisely $l$ steps in negative coordinate directions. We can associate a unique $n$-step path to $\gamma$ by removing these $l$ steps and the first $l$ compensating steps. Clearly, a

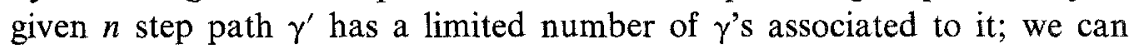
choose the $2 l$ steps removed in $\left(\begin{array}{c}n+2 l \\ 2 l\end{array}\right)$ ways and each such $2 l$ set has at most $(2 v)^{l}$ choices of directions, i.e.,

$$
N(\alpha, n+2 l) \leqslant\left(\begin{array}{c}
n+2 l \\
2 l
\end{array}\right)(2 v)^{l} N(\alpha, n)
$$

Using (12), we have

$$
\begin{aligned}
\sum_{\substack{2 l \geqslant n / 2 \\
k=n+2 l}} N(\alpha, k)[\tanh \beta]^{k} & \leqslant[\tanh \beta]^{n} \sum_{l=0}^{\infty}(2 \nu-1)^{6 l}(\tanh \beta)^{2 l} \\
& \leqslant 2 L(\alpha, \beta)
\end{aligned}
$$


so long as

$$
(2 v-1)^{6}(\tanh \beta)^{2} \leqslant 1 / 2
$$

On the other hand, if $2 l \leqslant n / 2$, then

$$
\left(\begin{array}{c}
n+2 l \\
2 l
\end{array}\right)=\left(\begin{array}{c}
n \\
2 l
\end{array}\right) \prod_{l=0}^{2 l-1} \frac{n+2 l-j}{n-j} \leqslant 3^{2 l}\left(\begin{array}{c}
n \\
2 l
\end{array}\right)
$$

(13) and (16) imply that

$$
\begin{aligned}
\sum_{\substack{2 l \leqslant n / 2 \\
k=n+2 l}} N(\alpha, k)[\tanh \beta]^{k} & \leqslant L(\alpha, \beta) \sum_{m=0}^{n}\left(\begin{array}{c}
n \\
m
\end{array}\right)(18 \nu)^{m / 2}[\tanh \beta]^{m} \\
& \leqslant L(\alpha, \beta)\left[1+(18 \nu)^{1 / 2}(\tanh \beta)\right]^{n}
\end{aligned}
$$

(14) and (17) imply the upper bound (5) whenever $\beta$ obeys (15).

\section{EXTENSIONS}

Let $d \mu_{0}$ have an arbitrary even probability measure of compact support on $(-\infty, \infty)$. Let

$$
a=\int x^{2} d \mu(x)
$$

One can consider an Ising-like model with nearest-neighbor of $\sigma_{i} \sigma_{j}$ interaction but with $\sum \sigma_{i}= \pm 1$ replaced by $\int \prod_{i} d \mu\left(\sigma_{i}\right)$. For this model, we claim that (1) holds if $\ln \beta$ is replaced by $\ln (a \beta)$. Paes-Leme ${ }^{(5)}$ obtains this result when $d \mu(x)=(2 s+1)^{-1} \sum_{1=-s}^{s} \delta(x-j)$ [although, for reasons that are unclear to me, he introduces two numbers $k_{1}$ and $k_{0}$ and writes $\ln \left(k_{1} \beta / k_{0}\right)$ with $k_{1}=a^{2}, k_{0}=a$ ].

To see that (1) holds in this modified form, one uses the diagrammatic expansion appropriate to such a model. In place of (6), one expands the exponential. Now diagrams have an integer $n_{b}$ associated to each bond $b$; for any site $\alpha, m_{\alpha}$ denotes the sum of the $n_{b}$ overbounds containing $\alpha$ and the value of a graph is

$$
\beta^{\sum n_{b}}\left(\prod_{b} n_{b} !\right)^{-1} \prod_{\alpha}\left[\int x^{m_{\alpha}} d \mu(x)\right]
$$

The expectation $\left\langle\sigma_{0} \sigma_{\alpha}\right\rangle_{\Lambda, \beta}$ is a ratio when the denominator is graphs of precisely this type (automatically the graph is zero unless all $m_{\alpha}$ are even) and the numerator has graphs of this type with exactly one bound from $\sigma_{0}$ to $\sigma_{\alpha}$ included.

The two critical properties (a) and (b) of spin-1/2 graphs also hold for these graphs. Since the lower bound really only depended on these 
properties, we immediately get the lower bound

$$
\begin{aligned}
\left\langle\sigma_{0} \sigma_{\alpha}\right\rangle_{\Lambda, \beta} \geqslant & \left(\alpha_{1}+\cdots+\alpha_{\nu}\right) !\left[\alpha_{1} ! \cdots \alpha_{\nu} !\right]^{-1} \beta^{\sum \alpha_{i}}(a)^{\sum \alpha_{i}+1} \\
& \times \exp \left[-\beta(2 \nu)\|\sigma\|^{2}\left(1+\sum \alpha_{i}\right)\right]
\end{aligned}
$$

where

$$
\|\sigma\|=\sup _{x}\{x \in \operatorname{support} \text { of } \mu\}=\lim _{n \rightarrow \infty}\left[\int x^{2 n} d \mu(x)\right]^{n / 2}
$$

As for the upper bound, we still have one in terms of general (i.e., not necessarily self-avoiding) random walks if we note that any connected graph with boundary $\{0, \alpha\}$ results from some random walk for 0 to $\alpha$ (an elementary combinatorial argument). If $(2 \nu-1)$ is replaced by $(2 \nu)$ in (12), the basic combinatorial estimates (12), (13), and (16) still hold. In estimating graphs, we ignore the helpful $\left[n_{b} !\right]^{-1}$ factors and use (if $m \geqslant 2$ )

$$
\left|\int x^{m} d \mu(x)\right| \leqslant a\|\sigma\|^{m-2}
$$

to obtain

$$
\left\langle\sigma_{0} \sigma_{\alpha}\right\rangle_{\Lambda, \beta} \leqslant 3 L(\alpha, \beta)\left[1+\|\sigma\|^{2}(12 v)^{1 / 2} \tanh \beta\right]^{\sum \alpha_{i}}
$$

so long as

$$
\|\sigma\|^{4}(2 \nu)^{6} \beta^{2} \leqslant 1 / 2
$$

where now

$$
L(\alpha, \beta)=\left(\alpha_{1}+\cdots+\alpha_{\nu}\right) !\left[\alpha_{1} ! \cdots \alpha_{\nu} !\right]^{-1}(\beta a)^{\sum \alpha_{j}} a
$$

These bounds imply the claimed general form of (1).

It also should be easy to extend these arguments for general finite range pair instructions.

\section{ACKNOWLEDGMENTS}

It is a pleasure to thank D. Bessis for telling me of Paes-Leme's paper and the Fairchild Visiting Scholar program for its hospitality.

\section{REFERENCES}

1. D. Abraham and H. Kunz, Phys. Rev. Lett. 39:1011 (1977).

2. W. J. Camp, Phys. Rev. 86:960 (1972).

3. W. J. Camp and M. Fisher, Phys. Rev. Lett. 26:73 (1971).

4. M. Fisher, Phys. Rev. 162:475 (1967).

5. P. J. Paes-Leme, Ann. Phys. (New York) 115:367 (1978). 